\title{
Contribuição das tecnologias da indústria 4.0 para a sustentabilidade: uma revisão sistemática
}

Contribution of industry 4.0 technologies to sustainability: a systematic review

\author{
Aline Xavier dos Santos Germano \\ Centro Federal de Educação Técnológica Celso Suckow da \\ Fonseca, Brasil \\ aline.santos@cefet-rj.br \\ (iD https://orcid.org/0000-0002-8081-9379
}

José André Villas Boas Mello

Centro Federal de Educação Técnológica Celso Suckow da

Fonseca, Brasil

jose.mello@cefet-ri.br

(iD https://orcid.org/0000-0002-0628-9664

Wladmir Henriques Motta

Centro Federal de Educação Técnológica Celso Suckow da

Fonseca, Brasil

wladmir.motta@cefet-ri.br

(D) https://orcid.org/0000-0002-8254-1278

\begin{abstract}
Resumo:
Este estudo trata se de uma revisão sistemática e tem como objetivo analisar como a pesquisa sobre a indústria 4.0 está contribuindo para um maior entendimento dos efeitos da implantação da indústria 4.0 na sustentabilidade. Este estudo foi feito mapeando a pesquisa já existente, utilizando critérios de seleção, elegibilidade e inclusão como eixo norteador da técnica da metodologia PRISMA (Preferred Reporting Items for Systematic Reviews and Meta-Analyses). A seleção dos artigos nas bases de dados consultadas foi baseada no cruzamento dos termos "Industry 4.0"e "Sustainability". A pesquisa traz como resultados os principais desafios para a implantação das tecnologias referentes à indústria 4.0, entre eles a falta de infraestrutura tecnológica e de recursos para investimento e quais os possíveis benefícios para a sustentabilidade que essa implantação pode trazer, como a redução no consumo de recursos em todas as fases da produção, bem como nos impactos ambientais gerados. Através deste estudo foi possível concluir que apesar de apresentar fatores que influenciam positivamente na sustentabilidade das empresas, a implantação das novas tecnologias para a adoção do conceito de indústria 4.0, ainda está cercada de riscos e desafios e existe a necessidade de novos estudos que explorem e consolidem os resultados referentes à quarta revolução industrial.
\end{abstract}

PaLAVRAS-CHAVE: Indústria 40, Sustentabilidade, Prisma, Quarta revolução industrial.

\section{Abstract:}

This study is a systematic review and aims to analyze how research on industry 4.0 is contributing to a greater understanding of the effects of the implementation of industry 4.0 on sustainability. This study was done by mapping the existing research, using selection, eligibility and inclusion criteria as the guiding principle of the PRISMA methodology technique (Preferred Reporting Items for Systematic Reviews and Meta-Analyses). The selection of articles in the databases consulted was based on the crossing of the terms "Industry 4.0" and "sustainability". The research brings as results the main challenges for the implementation of technologies related to industry 4.0, among them the lack of technological infrastructure and resources for investment and what the possible benefits for sustainability this implementation can bring, such as the reduction in the consumption of resources at all stages of production, as well as the environmental impacts generated. Through this study it was possible to conclude that despite presenting factors that positively influence the sustainability of companies, the implementation of new technologies for 
the adoption of the concept of industry 4.0, is still surrounded by risks and challenges and there is a need for further studies that explore and consolidate the results of the fourth industrial revolution.

KEYWORDS: Industry 4.0, Sustainability, Prism, Fourth industrial revolution.

\section{INTRODUÇÃo}

O termo "Indústria 4.0” surgiu na Alemanha no ano de 2011. “A quarta revolução industrial, também chamada de Indústria 4.0, foi gerada com tecnologias de informação e inteligência emergentes e disruptivas. Essas novas tecnologias estão permitindo níveis cada vez mais altos de eficiência de produção" (Bai et al., 2020). Dentre essas tecnologias pode-se citar a internet das coisas, computação em nuvem, impressão 3D, big data, entre outras (Pimenta, 2019). Segundo Nardo, Forino e Murino (2020), sistemas e máquinas inteligentes, caracterizam as novas fabricas denominadas "fábricas inteligentes".

Esses sistemas e máquinas se comunicam através da rede (Internet das coisas, IoT). Os dados vindos dos sistemas operacionais são coletados e analisados em tempo real. Esse monitoramento em tempo real permite que se possa realizar intervenções, quando por exemplo algum dispositivo ou máquina reage de forma inesperada e diferente do padrão estabelecido. Presume-se que a interconectividade promovida pela internet será superior a 26 bilhões de elementos/produtos já neste ano, fato este que promoverá ainda mais a utilização das tecnologias de Indústria 4.0 nesta nova era da manufatura (Bahrin, Otman, Azli e Talib, 2016; Botha, 2018; Saucedo-Martínez, Pérez Lara, Marmolejo-Saucedo, Salais-Fierro e Vasant, 2018; Hizam-Hanafiah e Soomro, 2021).

Atender à crescente demanda mundial por bens de capital e de consumo de forma sustentável é sem dúvida, um dos maiores desafios enfrentados pela sociedade atual. Sob a perspectiva de que as organizações buscam desenvolver estratégias que lhes permitam avançar para uma maior valorização dos produtos/ serviços prestados aos seus clientes (Requeijo, Abreu, Calado \& Dias 2018) e de que o tema indústria 4.0 tem sido objeto de pesquisa de diversos autores (Pimenta, 2019), pesquisadores da área de ciência da informação tem se dedicado a desenvolver revisões sistemáticas de literatura (RSL) como as de Kamble, Gunasekaran \& Gawankar (2018), Sima, Gheorge, Subic \& Nancu (2020) e Sony e Naik (2020), iniciativas que buscam compreender esse fenômeno e aglutinar respostas sobre esse tema. Diferentemente das RSL citadas, este estudo recai sobre o contexto da sustentabilidade, englobando suas três dimensões, econômica, social e ambiental, o que é visto como relevante por Bai, Dallasega, Orzes \& Sarkis (2020) que afirma que as organizações ainda precisam considerar a contribuição das tecnologias da Indústria 4.0 para a sustentabilidade. Tendo como perspectiva de que a indústria 4.0 pode ajudar neste processo, este estudo busca identificar os benefícios trazidos pela sua adoção, identificando também os possíveis desafios e demais impactos que sua implantação pode gerar.

Por se tratar de um tema relativamente novo, a intenção deste trabalho é reunir informações e resultados encontrados nos estudos já publicados, através de uma revisão sistemática, a fim de se chegar a indícios de como a Indústria 4.0 pode afetar a sustentabilidade das empresas. Além desta introdução o estudo apresenta uma seção de metodologia, uma seção de resultados, discussão e a conclusão.

\section{Metodologia}

A RSL é uma metodologia que busca fornecer aos especialistas um sistema que lhes permita obter com rapidez e eficiência os trabalhos mais relevantes sobre um tema (Mangas-Veja, Sánchez Jara \& Alonso, 2018; Ramírez, 2007). As RSL estruturadas auxiliam na condução das pesquisas que apresentam lacunas a serem exploradas (Cochrane e Mello, 2020; Rodrigues, Mello \& Alonso, 2019). A metodologia é apontada como a mais importante da pesquisa, pois define os meios pelos quais serão buscadas e obtidas as respostas aos 
problemas que ocorrem nas diferentes áreas do conhecimento. Evidências de revisão de meta-análise de vários estudos primários a partir do uso de instrumentos estatísticos combinados para aumentar a objetividade e a validade dos resultados (Concolato, Cunha \& Afonso, 2020; Dias, Reis, Oliveira, Maruyama \& Martínez, 2018).

Este estudo foi realizado utilizando-se critérios de seleção, elegibilidade e inclusão de estudos da técnica da metodologia PRISMA, que segundo Galvão, Pansani \& Harrad (2015) se baseia num checklist de 27 itens e num fluxograma de 4 etapas que apoiam os autores no relato das revisões sistemáticas e meta-análises. A recomendação PRISMA, foi desenvolvida através da reunião de um grupo com 29 participantes, formado por autores de revisões, editores, metodologistas, clínicos e um consumidor, que se reuniram no Canadá no ano de 2005, para revisar a recomendação QUOROM (Quality of Reporting of Meta-Analyses), que tinha como foco os relatos de meta-análises de ensaios clínicos randomizados. Dito isto, o estudo foi realizado seguindo as etapas a seguir.

\subsection{Pergunta orientadora}

A quarta revolução industrial pode potencialmente resolver muitas das limitações ecológicas e sociais das práticas e tecnologias industriais tradicionais; para fornecer um futuro mais sustentável (Morrar, Arman \& Mousa, 2017). Diante disto, o estudo apresenta uma questão norteadora, utilizada como guia para a busca de estudos em bases de dados pré-definidas. A questão principal utilizada para o início deste estudo foi: "Como a implantação da indústria 4.0 e suas tecnologias estão influenciando na sustentabilidade das empresas?". Com base neste questionamento o cruzamento dos termos "Industry 4.0" e "sustainability" foram escolhidos para a pesquisa de artigos nas bases de dados selecionadas.

\subsection{Bases de dados investigadas}

As bases de dados selecionadas para o levantamento dos artigos foram Web of Science, Scopus e Scielo que são consideradas referências mundiais. A busca foi realizada através do acesso CAFE (Comunidade Acadêmica Federada) disponível no Portal brasileiro de Periódicos CAPES/MEC. A busca pelos estudos foi realizada no dia 03 de setembro de 2020.

\subsection{Critérios de inclusão e exclusão}

Como critérios de inclusão e exclusão dos trabalhos encontrados foram utilizados:

- Publicações anteriores ao ano de 2015: foram excluídos todos os estudos publicados anteriormente ao ano de 2015, sendo utilizadas apenas as publicações mais recentes.

- Materiais que não sejam artigos científicos: nesta etapa foram incluídos, apenas os materiais que fossem artigos científicos, por apresentarem um processo de aprovação para publicação mais rigoroso.

- Acesso Fechado: foram eliminados todos os artigos de acesso fechado.

- Duplicatas: foram excluídos os materiais duplicados nas bases.

\subsection{Análise dos artigos elegíveis}

Para realizar a triagem final dos artigos, os estudos passaram por uma análise dos títulos e resumos, com o objetivo de identificar e eliminar os artigos que possuem um direcionamento diferente do objeto do presente 
estudo, sendo importante ressaltar que esta etapa é subjetiva e não se pode descartar a possibilidade de que algum dos artigos excluídos tenha relevância para o tema. Após essa eliminação prévia, os artigos restantes foram lidos na íntegra para a análise de elegibilidade. Estão incluídos na pesquisa, apenas os estudos que mais se aproximavam da temática pesquisada.

\section{Resultados}

\subsection{Principais itens para relatar revisões sistemáticas e meta - análises}

O levantamento utilizando as expressões "Industry 4.0" e "sustainability" nas bases de dados escolhidas teve como retorno, um total de 735 estudos. Após a utilização dos critérios de inclusão e exclusão tivemos os seguintes resultados: No critério "Publicações anteriores ao ano de 2015", foram eliminados apenas 8 artigos, no critério "materiais que não sejam artigos", foram eliminados um total de 352 materiais, como revisões sistemáticas, livros e capítulos de livros , no critério "Acesso fechado", foram eliminados 165 artigos, no critério "Duplicatas", foram retirados os artigos duplicados nas bases que somavam 69 artigos, restando apó ssta etapa 141 artigos, que foram utilizados para a elaboração do gráfico abaixo, que demonstra o crescimento da pesquisa sobre indústria 4.0 e sustentabilidade nos últimos anos. Podemos verificar claramente o crescimento da pesquisa sobre o tema e não podemos deixar de considerar que para o ano de 2020 foi considerado o dia 03 de setembro como data de corte e novas pesquisas poderão ser incluídas neste período.

FIGURA 1

Evolução anual das pesquisas que envolvem indústria 4.0 e sustentabilidade

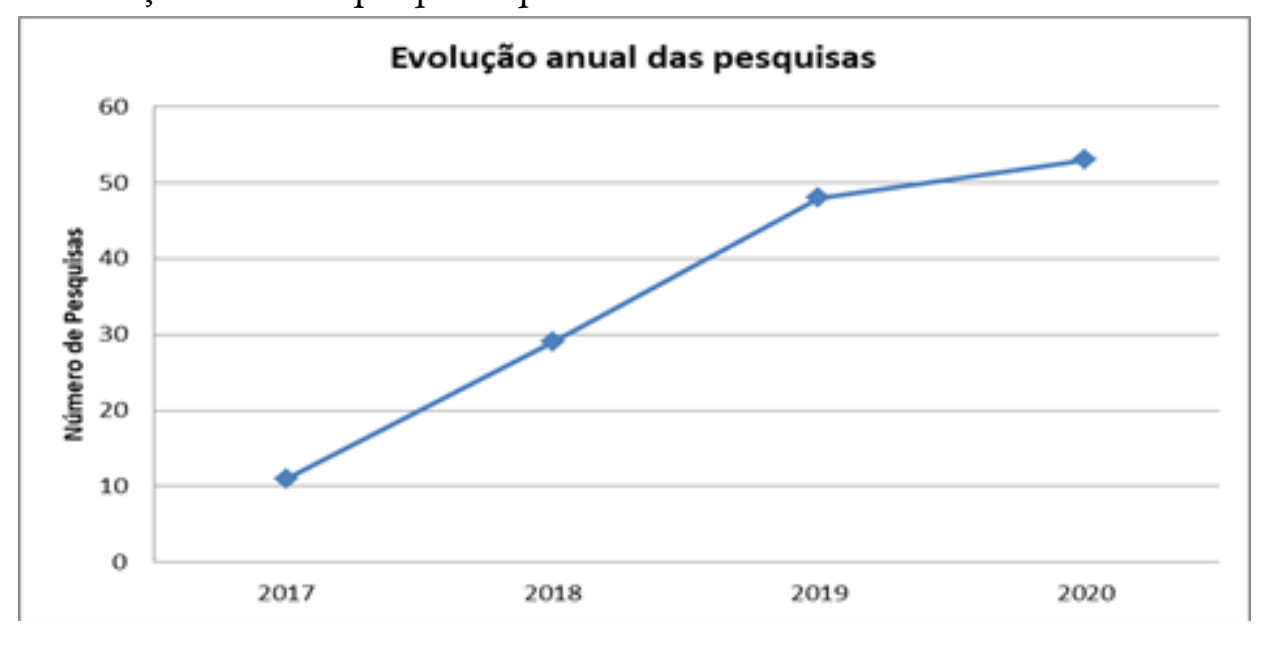

Fonte: elaborado pelos autores.

Por fim na análise dos títulos e resumos, para identificar e eliminar os artigos que possuíam um direcionamento diferente do objeto do presente estudo, foram eliminados 111 artigos. Restaram 30 artigos para a análise de elegibilidade. $\mathrm{Na}$ análise de elegibilidade, os artigos foram lidos na íntegra e 10 deles foram eliminados por não tratarem a fundo da questão pesquisada. Assim 20 artigos foram considerados para esta revisão sistemática. A figura 2 representa o fluxograma de seleção. 
FIGURA 2:

Fluxograma do processo de seleção dos estudos a partir da metodologia PRISMA

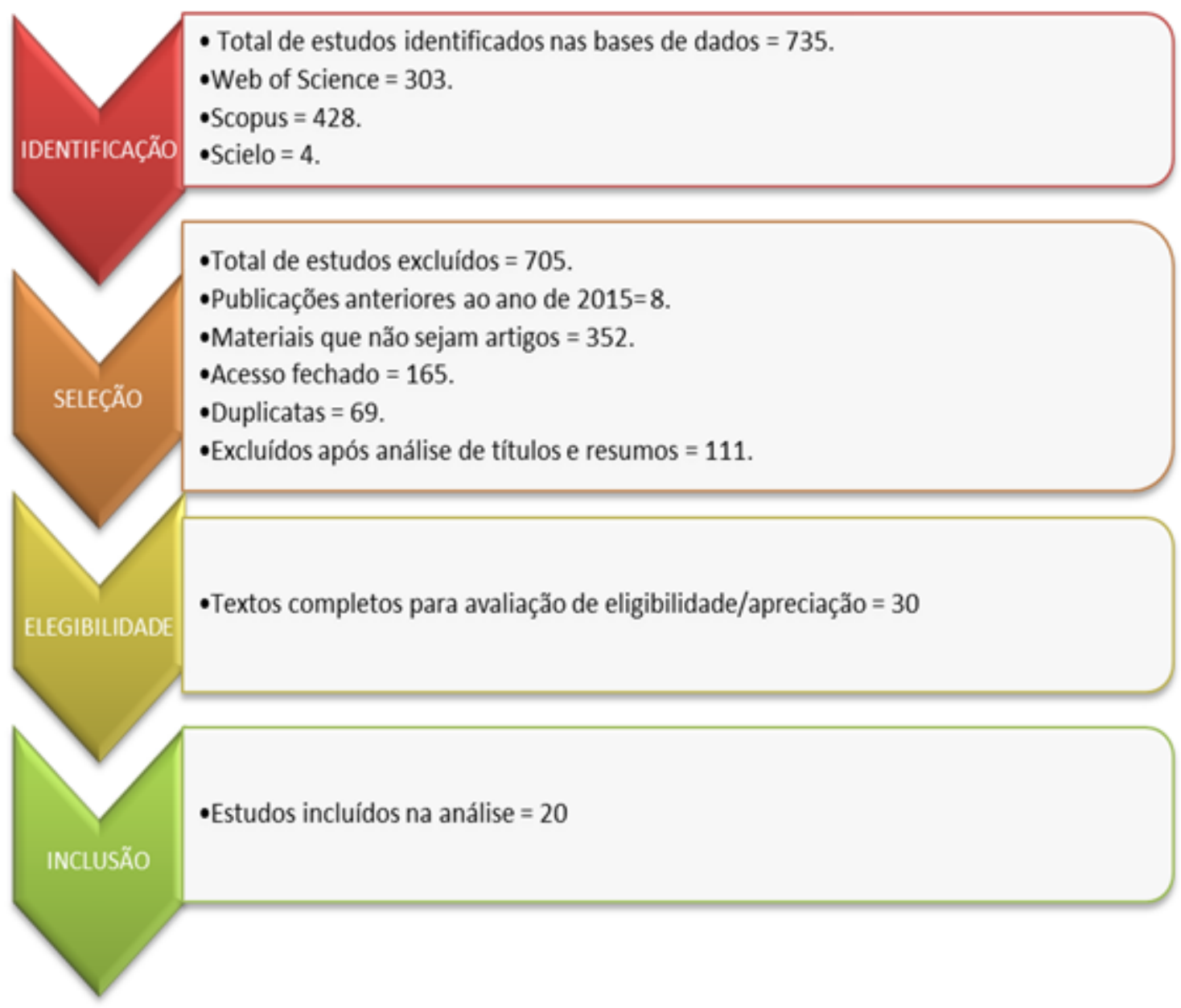

Fonte: elaborado pelos autores.

Uma nova análise de crescimento anual das pesquisas foi realizada, a fim de identificar se os estudos selecionados estavam seguindo o mesmo padrão e como ilustra o gráfico da figura 3, os estudos selecionados se mantiveram no mesmo padrão de crescimento.

FIGURA 3

Evolução anual das pesquisas selecionadas

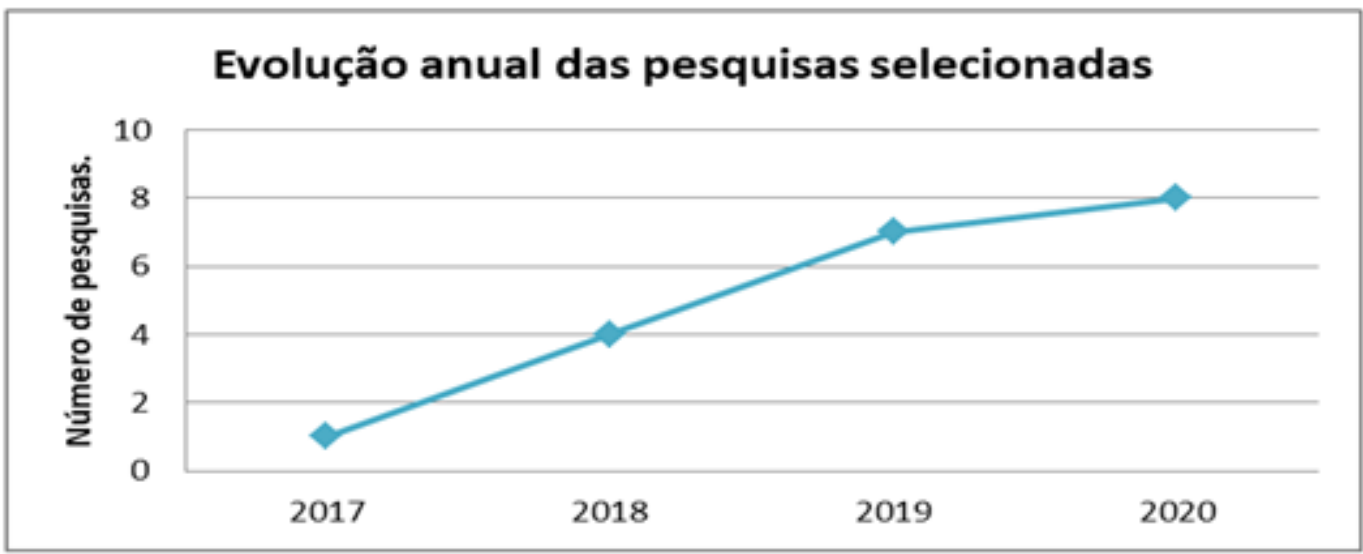

Fonte: elaborado pelos autores 


\subsection{Análise de palavras - chave}

Foi realizado o levantamento das palavras-chave utilizadas pelos autores dos 20 artigos selecionados, para identificar quais termos são os mais citados. Foram identificadas 79 palavras-chave diferentes. A figura 4 ilustra através de uma nuvem de palavras a representatividade de cada termo citado nas pesquisas analisadas. Os termos de maior destaque foram "Indústria 4.0" e "Sustentabilidade", como era o esperado, tendo em vista que os mesmos foram utilizados para a busca dos estudos nas bases de dados.

FIGURA 4:

Nuvem de palavras

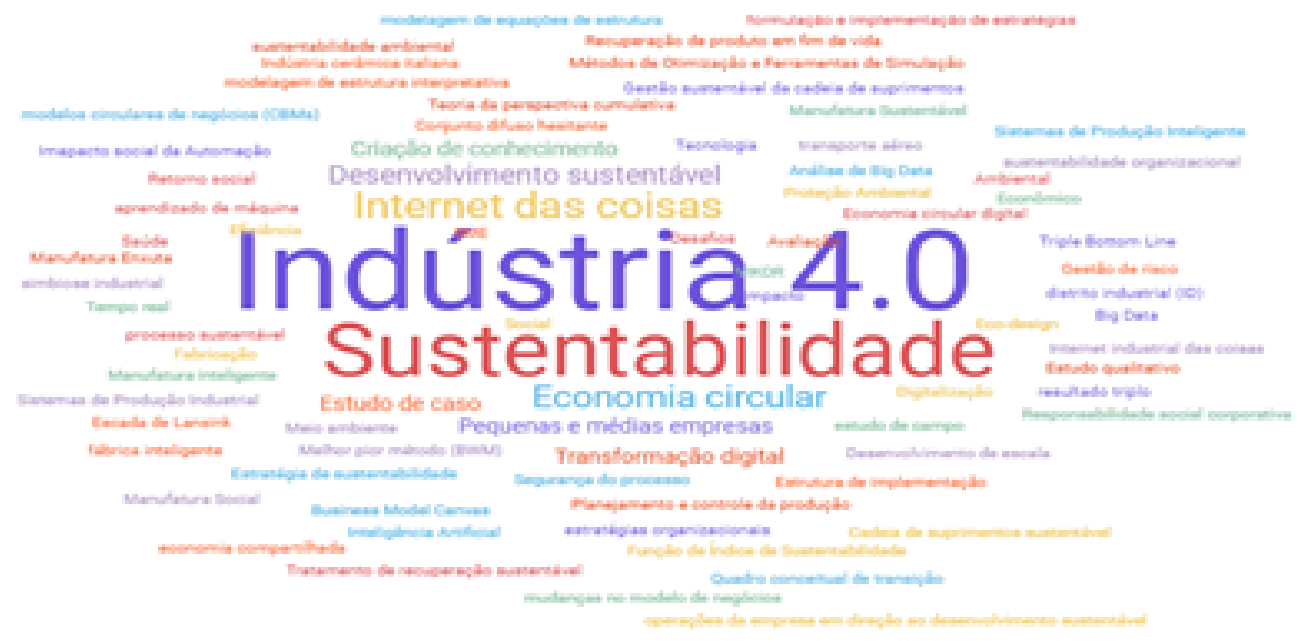

Fonte: elaborado pelos autores.

A tabela 1 apresenta as palavras mais citadas, juntamente com o número de vezes que foram registradas e o percentual de participação referente ao total de artigos. Para esta tabela foram consideradas apenas as palavras que alcançaram no mínimo $10 \%$ de participação nos artigos.

TABELA 1

Levantamento de palavras-chave

\begin{tabular}{lcc}
\hline \multicolumn{1}{c}{ Palavras -Chave Citadas } & $\begin{array}{c}\text { Número de } \\
\text { Registros }\end{array}$ & Percentual de participação \% \\
\hline \hline Indústria 4.0 & 18 & 90 \\
Sustentabilidade & 12 & 60 \\
Internet das coisas & 5 & 25 \\
Economia circular & 4 & 20 \\
Desenvolvimento sustentável & 3 & 15 \\
Pequenas e médias empresas & 2 & 10 \\
Estudo de caso & 2 & 10 \\
Transformação digital & 2 & 10 \\
Criação de conhecimento & 2 & 10 \\
\hline
\end{tabular}


A tabela 1 confirma o destaque evidenciado na nuvem de plavras dos termos “ indústria 4.0" e "sustentabilidade", porém, se pode notar a falta de termos importantes relacionados ao tema como, "Quarta Revolução Industrial” , que é um sinônimo de indústria 4.0 e não foi utilzado por nenhum dos autores, ou do termo "Big data", que é um das principais tecnologias utilizadas pela indústria 4.0 e foi utilizado em apenas um dos estudos, o que poderia resultar numa recuperação de textos mais eficiente, tendo em vista que muitos periódicos apresentam restrições com relação a escolha das plavras-chave, como por exemplo não utilizar palavras já escolhidas para o título. "A seleção de palavras-chave visa facilitar a recuperação eficiente do conteúdo de um texto para os leitores. Além disso, por serem ferramentas fundamentais para a indexação nas bases de dados, elas atuam como porta de acesso ao texto" (Waibel, Steenkamp, Moloko e Oosthuizen, 2017, pp. 731-737). Segundo os autores, a maioria dos autores subestimam a esta etapa da pesquisa, não fazendo uma reflexão mais aprofundada sobre as melhores opções e isso reduz significativamente a possibilidade de localização dos seus documentos, acarretando na sedimentação dos seus textos nas grandes bases.

\subsection{Localização dos estudos}

Foi feita uma análise para verificar como as publicações analisadas estão distribuídas com relação ao local onde foram realizadas. As tabelas 2 e 3 foram geradas a partir dos resultados desta análise e como podemos ver na tabela 2 a maioria dos estudos foi realizada na Europa, com cerca de $65 \%$ das publicações, tendo a Itália com líder do ranking com 4 registros (tabela3). A sia está em segundo lugar, mas com um número de registro bem inferior ao da Europa, o que representa apenas 15\% dos estudos analisados. Em seguida temos a América do Sul, que de acordo com os dados é representada apenas pelo Brasil e apesenta apenas 10\% das pesquisas. Finalmente temos a frica e América do norte com apenas 5\% dos estudos cada.

TABELA 2:

Distribuição dos estudos analisados por continentes

\begin{tabular}{ccc}
\hline Continente & Registros & Percentual \\
\hline \hline Europa & 13 & $65 \%$ \\
Ásia & 3 & $15 \%$ \\
\hline América do Sul & 2 & $10 \%$ \\
África & 1 & $5 \%$ \\
América do Norte & 1 & $5 \%$ \\
\hline
\end{tabular}

Fonte: elaborado pelos autores 
TABELA 3:

Distribuição dos estudos analisados por países:

\begin{tabular}{ccc}
\hline $\begin{array}{c}\text { Origem da } \\
\text { Pesquisa }\end{array}$ & Registros & Percentual \\
\hline \hline Itália & 4 & $20 \%$ \\
\hline Alemanha & 3 & $15 \%$ \\
\hline Espanha & 2 & $10 \%$ \\
\hline China & 2 & $10 \%$ \\
\hline Brasil & 2 & $10 \%$ \\
\hline Hungria & 1 & $5 \%$ \\
\hline Noruega & 1 & $5 \%$ \\
\hline Portugal & 1 & $5 \%$ \\
\hline Romênia & 1 & $5 \%$ \\
Bangladesh & 1 & $5 \%$ \\
\hline África do Sul & 1 & $5 \%$ \\
Canadá & 1 & $5 \%$ \\
\hline
\end{tabular}

Fonte: elaborado pelos autores.

\subsection{Distribuição dos estudos em periódicos}

Os resultados dos periódicos que mais publicaram os estudos analisados foram incluídos na tabela 3, com a intensão de facilitar a busca de novos pesquisadores interessados no tema proposto. O periódico que mais publicou os artigos utilizados para a presente pesquisa foi a revista Sustainability.

TABELA 4

Relação de periódicos que mais publicaram

\begin{tabular}{cc}
\hline Periódico & Número de Registros \\
\hline \hline Sustainability (Switzerland) & 12 \\
IFAC-Papers OnLine & 2 \\
Journal of Business Research & 1 \\
International Journal of Production Economics & 1 \\
Procedia Manufacturing & 1 \\
Process Safety and Environmental & 1 \\
Processes & 1 \\
Social Sciences & 1 \\
\hline
\end{tabular}

Fonte: elaborado pelos autores.

\subsection{Foco dos estudos analisados}

O quadro 1 a seguir, apresenta os autores e os títulos de todos os estudos analisados nesta pesquisa. Todos os estudos selecionados foram publicados em inglês. 
QUADRO 1

Identificação dos estudos

\begin{tabular}{|c|c|c|}
\hline Autores & Título & Foco dos estudos \\
\hline Molkadir et al. & $\begin{array}{l}\text { Assessing challenges for } \\
\text { implementing Industry } 4.0 \text { : } \\
\text { Implications for process } \\
\text { safety and environmental } \\
\text { protection }\end{array}$ & $\begin{array}{l}\text { O artigo aborda os maiores desafios para a } \\
\text { implementação da indústria } 4.0 \text { nas manufaturas, } \\
\text { visando a avaliação e conhecimento prévio dos } \\
\text { mesmos, para alcançar uma estratégia assertiva. Foco } \\
\text { na indústria de couro de Bangladesh. }\end{array}$ \\
\hline Bai et al. & $\begin{array}{l}\text { Industry } 4.0 \text { technologies } \\
\text { assessment: A sustainability } \\
\text { perspective }\end{array}$ & $\begin{array}{l}\text { O estudo argumenta que a implementação da } \\
\text { indústria } 4.0 \text { vai além do viés econômico e traz uma } \\
\text { contribuição para uma sociedade mais sustentável. } \\
\text { Mostra os desafios da implementação com foco na } \\
\text { sustentabilidade. }\end{array}$ \\
\hline $\begin{array}{l}\text { Kristoffersen, } \\
\text { et.al. }\end{array}$ & $\begin{array}{l}\text { The smart circular economy: } \\
\text { A digital-enabled circular } \\
\text { strategies framework for } \\
\text { manufacturing companies }\end{array}$ & $\begin{array}{l}\text { O estudo aborda a ligação entre as tecnologias digitais } \\
\text { e a economia circular e como aproveitar as } \\
\text { tecnologias digitais para maximizar a eficiência dos } \\
\text { recursos e a produtividade para uma estratégia } \\
\text { circular específica. }\end{array}$ \\
\hline Autores & Título & Foco dos estudos \\
\hline Oláh et al. & $\begin{array}{l}\text { Impact of industry } 4.0 \text { on } \\
\text { environmental sustainability }\end{array}$ & $\begin{array}{l}\text { O estudo traz uma discussão sobre os impactos da } \\
\text { indústria } 4.0 \text { na sustentabilidade ambiental, avaliando } \\
\text { as possíveis vantagens e desvantagens a curto e a } \\
\text { longo prazo. }\end{array}$ \\
\hline Brozzi et al. & $\begin{array}{l}\text { The advantages of industry } \\
4.0 \text { applications for } \\
\text { sustainability: Results from a } \\
\text { sample of manufacturing } \\
\text { companies }\end{array}$ & $\begin{array}{l}\text { O estudo busca saber se as empresas de manufatura } \\
\text { percebem na implantação da indústria } 4.0 \\
\text { oportunidades de sustentabilidade ambiental e social, } \\
\text { além de investigar as vantagens da implementação } \\
\text { para a sustentabilidade. }\end{array}$ \\
\hline Ivascu, L. & $\begin{array}{l}\text { Measuring the implications of } \\
\text { sustainable manufacturing in } \\
\text { the context of industry } 4.0\end{array}$ & $\begin{array}{l}\text { O estudo faz um levantamento das características da } \\
\text { Indústria } 4.0 \text { e dos indicadores que avaliam a } \\
\text { sustentabilidade organizacional, afim de montar uma } \\
\text { estrutura para a avaliação da sustentabilidade na } \\
\text { manufatura. }\end{array}$ \\
\hline Oluyisola et al. & $\begin{array}{l}\text { Smart production planning } \\
\text { and control: Concept, use- } \\
\text { cases and sustainability } \\
\text { implications }\end{array}$ & $\begin{array}{l}\text { O e studo faz uma abordagem prática da implantação } \\
\text { da indústria } 4.0 \text { no controle e planejamento da } \\
\text { produção(PPC) e suas implicações na } \\
\text { sustentabilidade, utilizando estudos de caso reais } \\
\text { como base. }\end{array}$ \\
\hline $\begin{array}{l}\text { Garcia-Muiña et } \\
\text { al. }\end{array}$ & $\begin{array}{c}\text { Sustainability transition in } \\
\text { industry } 4.0 \text { and smart } \\
\text { manufacturing with the triple- } \\
\text { layered business model } \\
\text { canvas }\end{array}$ & $\begin{array}{l}\text { O estudo tem como objetivo fazer uma análise da } \\
\text { migração de um modelo de negócios tradicional para } \\
\text { um modelo de negócios sustentável. Essa migração } \\
\text { foi feita por meio de investimentos em tecnologias da } \\
\text { indústria 4.0. }\end{array}$ \\
\hline $\begin{array}{l}\text { Ordieres-Meré, } \\
\text { J., Remón, T.P., } \\
\text { Rubio, J. }\end{array}$ & $\begin{array}{c}\text { Digitalization: An opportunity } \\
\text { for contributing to } \\
\text { sustainability from knowledge } \\
\text { creation }\end{array}$ & $\begin{array}{l}\text { O estudo tem como proposta, analisar quais os efeitos } \\
\text { para a sustentabilidade, quando a transformação } \\
\text { digital, impulsionada pela indústria } 4.0 \text { é adotada por } \\
\text { uma organização. }\end{array}$ \\
\hline Pham et al. & $\begin{array}{l}\text { Industry } 4.0 \text { to accelerate the } \\
\text { circular economy: A case } \\
\text { study of electric scooter } \\
\text { sharing }\end{array}$ & $\begin{array}{l}\text { O artigo explora os conceitos fundamentais da } \\
\text { Indústria } 4.0 \text { e os fatores influentes da Indústria } 4.0 \\
\text { que podem contribuir para a quebra de barreiras } \\
\text { enfrentadas para a implantação da Economia } \\
\text { Circular. }\end{array}$ \\
\hline Naderi et al. & $\begin{array}{l}\text { Sustainable operations } \\
\text { management for industry } 4.0 \\
\text { and its social return }\end{array}$ & $\begin{array}{l}\text { O e studo tem como objetivo o desenvolvimento de } \\
\text { ferramentas e procedimentos para a digitalização em } \\
\text { PMEs, utilizando tecnologias da indústria } 4.0 \text { visando } \\
\text { a sustentabilidade. }\end{array}$ \\
\hline $\begin{array}{l}\text { Keivanpour, } S ., \\
\text { Ait Kadi, D. }\end{array}$ & $\begin{array}{l}\text { Internet of things enabled } \\
\text { real-time sustainable end-of- } \\
\text { life product recovery }\end{array}$ & $\begin{array}{l}\text { O artigo tem como objetivo avaliar o impacto da loT, } \\
\text { do processamento de dados em tempo real e } \\
\text { tecnologias presentes na indústria } 4.0 \text {, na recuperação } \\
\text { do produto em fim de vida. }\end{array}$ \\
\hline $\begin{array}{l}\text { Garcia-Muiña et } \\
\text { al. }\end{array}$ & $\begin{array}{l}\text { Identifying the equilibrium } \\
\text { point between sustainability } \\
\text { goals and circular economy } \\
\text { practices in an Industry } 4.0 \\
\text { manufacturing context using } \\
\text { eco-design }\end{array}$ & $\begin{array}{l}\text { O estudo tem como meta, encontrar o equilíbrio entre } \\
\text { sustentabilidade e economia circular, utilizando como } \\
\text { ferramenta principal o eco-design. Além de } \\
\text { demonstrar como as tecnologias referentes à } \\
\text { indústria } 4.0 \text { podem ser utilizadas como fatores de } \\
\text { habilitação para este objetivo. }\end{array}$ \\
\hline Varela et al. & $\begin{array}{c}\text { Evaluation of the relation } \\
\text { between lean manufacturing, } \\
\text { industry } 4.0 \text {, and } \\
\text { sustainability }\end{array}$ & $\begin{array}{l}\text { O estudo busca identificar qual a influência do Lean } \\
\text { Manufacturing e da indústria } 4.0 \text { na sustentabilidade } \\
\text { nos seus três pilares, social ,econômico e ambiental e } \\
\text { a partir disso dar apoio a tomada de decisão as } \\
\text { empresas industriais e seus stakeholders. }\end{array}$ \\
\hline Birkel et al. & $\begin{array}{l}\text { Development of a risk } \\
\text { framework for Industry } 4.0 \text { in } \\
\text { the context of sustainability } \\
\text { for established manufacturers }\end{array}$ & $\begin{array}{l}\text { O artigo propõe o levantamento, a análise e discussão } \\
\text { dos riscos que envolvem a implementação da indústria } \\
4.0 \text {, no que diz respeito à sustentabilidade e se seus } \\
\text { três pilares, econômico, social e ambiental. }\end{array}$ \\
\hline
\end{tabular}




\begin{tabular}{|c|c|c|}
\hline Autores & Título & Foco dos estudos \\
\hline Daú et al. & $\begin{array}{c}\text { The healthcare sustainable } \\
\text { supply chain 4.0: The circular } \\
\text { economy transition } \\
\text { conceptual framework with } \\
\text { the corporate social } \\
\text { responsibility mirror }\end{array}$ & $\begin{array}{l}\text { O estudo tem como objetivo analisar a cadeia de } \\
\text { suprimentos sustentável em saúde e propor } \\
\text { arcabouço conceitual para a transição da economia } \\
\text { circular Diante deste objetivo entender como a } \\
\text { indústria } 4.0 \text { influencia a cadeia de abastecimento } \\
\text { circular. }\end{array}$ \\
\hline $\begin{array}{l}\text { Braccini, A.M., } \\
\text { Margherita, E.G. }\end{array}$ & $\begin{array}{l}\text { Exploring organizational } \\
\text { sustainability of Industry } 4.0 \\
\text { under the triple bottom line: } \\
\text { The case of a manufacturing } \\
\text { company }\end{array}$ & $\begin{array}{l}\text { O e studo tem como objetivo responder como a } \\
\text { indústria } 4.0 \text { apoia as dimensões de sustentabilidade } \\
\text { do triple bottom line (TBL) - ecológico, social e } \\
\text { econômico e quais as interações entre essas } \\
\text { dimensốes a indústria } 4.0 \text { suporta. }\end{array}$ \\
\hline Bonilla et al. & $\begin{array}{c}\text { Industry } 4.0 \text { and sustainability } \\
\text { implications: A scenario- } \\
\text { based analysis of the impacts } \\
\text { and challenges }\end{array}$ & $\begin{array}{l}\text { O artigo busca discutir o impacto da sustentabilidade e } \\
\text { os desafios da Indústria } 4.0 \text { a partir de quatro cenários } \\
\text { diferentes: implantação, operação e tecnologias, } \\
\text { integração e conformidade com as metas de } \\
\text { desenvolvimento sustentável e cenários de longo } \\
\text { prazo. }\end{array}$ \\
\hline $\begin{array}{l}\text { Müller, J.M., } \\
\text { Kiel, D., Voigt, } \\
\text { K.-I. }\end{array}$ & $\begin{array}{c}\text { What drives the } \\
\text { implementation of Industry } \\
4.0 \text { ? The role of opportunities } \\
\text { and challenges in the context } \\
\text { of sustainability }\end{array}$ & $\begin{array}{l}\text { O estudo tem como objetivo analisar a relevância das } \\
\text { oportunidade e desafios da implementação da } \\
\text { Indústria 4.0 como impulsionadores para a } \\
\text { implementação da Indústria } 4.0 \text { no contexto da } \\
\text { sustentabilidade }\end{array}$ \\
\hline Waibel et al. & $\begin{array}{l}\text { Investigating the Effects of } \\
\text { Smart Production Systems on } \\
\text { Sustainability Elements }\end{array}$ & $\begin{array}{l}\text { O estudo busca através de estudos de caso, investigar } \\
\text { a dinâmica da Indústria } 4.0 \text { e identificar os pré- } \\
\text { requisitos para as empresas utilizarem a produção } \\
\text { inteligente. }\end{array}$ \\
\hline
\end{tabular}

Fonte: elaborado pelos autores.

\section{Discussão}

Após a análise do conteúdo e leitura dos artigos foram identificados os tópicos mais importantes para discussão.

\subsection{Perfil da pesquisa sobre indústria 4.0 e sustentabilidade}

A sustentabilidade para ser efetiva deve atender aos interesses, social, econômico e ambiental. Segundo Horbac (2005) os três tipos de interesses que devem ser cumpridos de forma simultnea são o da geração atual em melhorar as suas condições de vida, representando a sustentabilidade econômica, o da equalização das condições de vida entre ricos e pobres, representando a sustentabilidade social, e a do interesse das gerações futuras que não estão comprometidas pela satisfação das necessidades da geração atual, representando a sustentabilidade ambiental.

No meio empresarial, a sustentabilidade foi introduzida pelo conceito de gestão sustentável, especificadamente em como as empresas produzem seus produtos e serviços, mantém e melhoram os recursos humanos e naturais (Sartori, Latronica \& Campos, 2014). O conceito de "Indústria 4.0" é relativamente novo, ele teve sua origem na Alemanha em 2011. Deste ano em diante o interesse pelo o assunto, despontou entre os pesquisadores. Neste estudo foram utilizados apenas os estudos publicados a partir de 2015, a fim de captarmos o que há de mais recente na área de pesquisa. Como podemos confirmar no gráfico presente na figura 1, há um crescimento contínuo nas pesquisas relacionando a indústria 4.0 à sustentabilidade.

De acordo com a análise dos estudos selecionados a Europa lidera as pesquisas na área, somando 65\% do total de pesquisa, um percentual maior do que o somatório de todas as outras regióes. Os estudos de 
caso realizados em empresas que já implantaram as tecnologias referentes à indústria 4.0, que constam nesta pesquisa, foram realizados em sua maioria em indústrias localizadas na Europa, o que parece evidenciar um avanço maior desta região sobre o restante do mundo no que toca a adoção e implantação da indústria 4.0.

Apesar do evidente crescimento das pesquisas, a escassez de pesquisa na área é citada por diversos autores. "Na Indústria 4.0, também existem barreiras e problemas que ainda requerem extensa pesquisa". (Muller, Kiel \& Voigt, 2018, p. 247). "Uma limitação importante da existência de pesquisas na indústria 4.0, suas tecnologias e sua aplicabilidade, é o conteúdo empírico limitado” (Oluyisola, Sgarbossa \& Strandhagen, 2020).

\subsection{Desafios da adoção da indústria 4.0 para a sustentabilidade}

Por ser um conceito recente, a implantação da indústria 4.0, ainda apresenta um rol extenso de riscos e desafios que ainda não são amplamente conhecidos, principalmente quando falamos de sustentabilidade. "Os efeitos reais da Indústria 4.0 na dimensão da sustentabilidade ainda são incertos e pouca atenção tem sido dada aos riscos em particular" (Pham et al., 2019, p. 6661) "Na Indústria 4.0, também existem barreiras e problemas que ainda requerem extensa pesquisa. Essas barreiras incluem a viabilidade cultural dos modelos propostos e o apoio necessário dos governos" (Bonilla, Silva, Terra da Silva, Goncalves \& Sacomano, 2018, p. 3740).

Em seu estudo, Moktadir, Ali, Kusi-Sapong \& Shaikh (2018), apresentam uma estrutura para analisar os principais desafios para implantação da indústria 4.0, utilizando o método de tomada de decisão multicritério chamado denominado Best Worst Method (BWM). A estrutura identifica desafios como necessidade de alto investimento, diminuição das oportunidades de trabalho, falta da estratégia para a indústria 4.0, efeitos colaterais ambientais, complexidade na reconfiguração do padrão de produção e falta de equipe de gestão qualificada, mas a falta de infraestrutura tecnológica é vista como a o maior desafio entre os citados, com base no questionamento feito aos responsáveis por empresas que sofreram a implantação. Na pesquisa realizada por Pham et al. (2019), os autores concluem que coleta e análise de dados, está entre as principais barreiras, o que também pode ser visto como a falta de infraestrutura tecnológica, citada por Moktadir, et al. (2018), também citam leis inflexíveis ou desatualizadas, limitações sócio-culturais entre as principais barreiras. Dalenogare, Benítez, Ayala \& Frank (2018), concluíram que as empresas, principalmente as dos países em desenvolvimento, preferem investir nas tecnologias de baixo custo como, tecnologia de rastreamento e gerenciamento do que no conjunto completo de ferramentas de suporte de dados. O que acaba criando uma situação em que os dados são coletados, mas não de forma que fomentem a tomada de decisão.

Um desafio que também é bastante citado é a questão da sustentabilidade ambiental, pois ainda não há um consenso entre os pesquisadores de que se os benefícios para o meio ambiente trazidos pela adoção da indústria 4.0, superam os possíveis impactos ambientais que sua implantação pode gerar, como por exemplo, o alto uso de matérias-primas na produção do novo maquinário necessário para a implantação da tecnologia da indústria 4.0, o descarte do maquinário obsoleto e o alto consumo de energia demandado por toda essa nova tecnologia. Kristoffersen, Blomsma, Mikalef \& Li (2020) abordam essa questão da troca de máquinas obsoletas, produção de novo maquinário, consumo de energia e matéria - prima e concluem que, para evitar um impacto ambiental negativo a reciclagem deve estar envolvida nesse processo, o que acarretará numa maior economia energética, trazendo uma solução que beneficiaria os gerentes de fábrica, o governo e as organizações e garantiria a sustentabilidade ambiental. Segundo Wang, Wa, Li \& Zhang (2016) para estar voltada para a sustentabilidade a indústria precisa ter sua produção de acordo com as restrições ambientais e a maior pressão se refere a crescente demanda de energia.

Do ponto de vista social, verificamos a preocupação de alguns autores que relatam que uma vez que a nova tecnologia empregada substitui funções que hoje são praticadas pelo homem, isso geraria um grande número de demissóes e como Braccini e Margherita (2018) citam em seu estudo, parte da força de trabalho passaria para o maquinário eliminando vários cargos. 


\subsection{Principais benefícios da implantação da indústria 4.0 para a sustentabilidade}

A mesma questão sobre o fato de ser um conceito recente e ainda ter muitos questionamentos a serem respondidos, se aplica com relação aos benefícios da indústria 4.0 para a sustentabilidade. "A nova evolução do processo produtivo e industrial denominado Indústria 4.0 e suas tecnologias relacionadas, como a Internet das coisas, big data analytics e sistemas ciberfísicos, entre outros, ainda têm um impacto potencial desconhecido sobre a sustentabilidade" (Bonilla et al., 2018, p. 3740).

Apesar de ainda não serem totalmente explorados, as pesquisas e literaturas já existentes, trazem a tona os principais benefícios para a sustentabilidade que pode se obter como resultado da implantação da indústria 4.0. Braccini e Margherita (2018) e Brozzi, Forti, Rauch \& Matt (2020), trazem em seu estudo que a adoção da indústria 4.0 pode trazer benefícios sociais, melhorando a produtividade e organizacional e também o ambiente de trabalho, já que parte do trabalho mais pesado é passado para as máquinas, o que expõe o trabalhador a um menor risco. Além disso, também destacam a flexibilidade de produção e uso eficiente de recursos como benefícios. Müller et al. (2018) levantaram em seu estudo como benefícios sociais, a possiblidade de maior flexibilização das funções corporativas através da digitalização, como a implantação do home office e outras abordagens do tele trabalho, a transferência de tarefas monótonas e repetitivas para os sistemas de produção inteligentes, trazendo assim, maior motivação e satisfação para os funcionários e até mesmo a preservação da saúde do trabalhador, através da implantação de dispositivos inteligentes em estações de trabalho que exijam muito fisicamente do colaborador, o que vai de encontro ao que foi citado por Braccini e Margherita (2018). Ordieres-Meré et al. (2020) identificam também a oportunidade de um maior compartilhamento de conhecimento entre os colaboradores, através do desenvolvimento da indústria 4.0 e da introdução de tecnologias que tornam mais eficiente o compartilhamento de informações como uma vantagem.

Como se vê nas pesquisas de Garcia-Muiña, Medina-Salgado, Ferrari, Volpi, Pini \& Settembre Blundo (2019), Ivascu (2020) e Keivanpour e Kadi (2019) um dos benefícios ambientais mais citados é a economia de recursos que pode ser obtida através do monitoramento em tempo real de todas as fases da produção. García-Muiña, Medina-Salgado, Ferrari \& Cucchi (2020) destacam a importância dos sensores ligados ao sistema de gestão que são capazes de identificar uma falha na produção e interromper o processo para evitar desperdício de matéria-prima e também de energia, viabilizando uma melhor análise dos impactos ambientais. De acordo com Bai, Dallasega, Orzes \& Sarkis (2020) e Varela, Araújo, Ávila, Castro \& Putnik (2019), as tecnologias da indústria 4.0 podem do ponto de vista ecológico, reduzir o consumo de energia e recursos nos processos de produção e cadeia de suprimentos e também serem utilizadas nas análises de pegada de carbono, podendo levar a uma possível redução de resíduos e emissões de CO2. Para Moktadir et al. (2018) e Oláh, Aburumman, Popp, Khan, Haddad \& Kitukutha (2020) as tecnologias mais limpas provenientes da indústria 4.0, empregados em processos de produção inteligentes, podem minimizar o desperdício e impacto ambiental negativo.

\section{CONCLUSÃO}

A adoção das novas tecnologias envolvidas na implantação da indústria 4.0, podem trazer grandes benefícios para a sustentabilidade que envolve aspectos econômicos sociais e ambientais, como o avanço na segurança e no cuidado com a saúde do trabalhador, economia de recursos, energia e emissão de gases poluentes, porém não se pode menosprezar os riscos e desafios trazidos pela quarta revolução industrial. Como foi levantado por alguns autores é preciso dar uma atenção especial para a infraestrutura tecnológica, tendo em vista que a falta da mesma é uma das principais barreiras para a implantação da indústria 4.0 com sucesso e também aos possíveis danos ambientais que sua adoção pode causar se não for bem administrada. 
A questão que levanta a escassez de pesquisa que relacione a indústria 4.0 e a sustentabilidade é altamente mencionada. Por ser um conceito relativamente novo e ainda estar se consolidando, se pode considerar que a pesquisa na área ainda se encontra em fase embrionária. Os estudos de caso que apresentam os reais resultados da implantação da indústria 4.0, são limitados, pois se referem a pequenas regióes e pequenos nichos da manufatura. Com isso, mesmo com a forte indicação de bons resultados, referentes à adoção das novas tecnologias que compõe a indústria 4.0, a comprovação desses benefícios ainda necessita de maior sustentação. Desta forma se pode enxergar que ainda existe uma enorme lacuna para ser explorada por novos estudos ao que se refere aos impactos da indústria 4.0 na sustentabilidade, principalmente no que toca a exploração das experiências de implantação já concretizadas, para que se tenha acesso a resultados reais e que o conhecimento desses resultados possibilitem tanto a tomada de decisão mais segura das organizações que estudam a possibilidade de se adaptar a esse novo contexto, quanto o apoio à criação de políticas públicas que favoreçam o melhor cenário, pelos governantes.

\section{REFERÊNCIAS}

Bahrin, M. A.K.; Othman, M. F.; Azli, N. H. N. \& Talib, M.F. (2016). Industry 4.0: A review on industrial automation and robotic.Jurnal teknologi, 78, 137-143. https://doi.org/10.11113/jt.v78.9285

Bai, C.; Dallasega, P.; Orzes, G. \& Sarkis, J. (2020). Industry 4.0 technologies assessment: A sustainability perspective. International journal of production economics, 229, 107776. http://dx.doi.org/10.1016/j.ijpe.2020.107776

Bonilla, S.; Silva, H.; Terra Da Silva, M.; Franco Gonçalves, R. \& Sacomano, J. (2018). Industry 4.0 and sustainability implications: a scenario-based analysis of the impacts and challenges. Sustainability, 10, 3740. http://dx.doi.or $\mathrm{g} / 10.3390 / \mathrm{su} 10103740$

Botha, A. P. (2018). Rapidly arriving futures: future readiness for industry 4.0. South African journal of industrial engineering, 29(3), 148-160. https://doi.org/10.7166/29-3-2056

Braccini, A. \& Margherita, E. (2018). Exploring organizational sustainability of industry 4.0 under the triple bottom line: The case of a manufacturing company. Sustainability, 11,36. http://dx.doi.org/10.3390/su11010036

Brozzi, R.; Forti, D.; Rauch, E. \& Matt, D. T. (2020). The advantages of industry 4.0 applications for sustainability: Results from a sample of manufacturing companies. Sustainability, 12, 3647. http://dx.doi.org/10.3390/su12 093647

Cochrane, D. R. R. \& Mello, J. A. V. B. (2020). Consequências da economia GIG na identidade profissional: Revisão sistemática da literatura. Logeion: filosofia da informação, 6(2), 142-156. https://doi.org/10.21728/logeion.202 0v6n2.p142-156

Concolato, C. O. F.; Cunha, M. R. \& Afonso, H. C. A. G. (2020). Economic feasibility for photovoltaic solar energy projects: a systematic review. Revista produção e desenvolvimento, 6. https://doi.org/10.32358/rpd.2020.v6.506

Dalenogare, L. S.; Benitez, G. B.; Ayala, N. F. \& Frank, A.G. (2018).The expected contribution of Industry 4.0 technologies for industrial performance. International Journal of production economics, 204, 383-394. http://d x.doi.org/10.1016/j.ijpe.2018.08.019

Dias, A. C.; Reis, A. C.; Oliveira, R. P.; Maruyama, U. \& Martinez, P. (2018). Lean manufacturing in healthcare: A systematic review of literature. Revista produção e desenvolvimento, 4(2), 111-122. http://dx.doi.org/10.32358 /rpd.2018.v4.285

Galvão, T. F.; Pansani, T. S. A. \& Harrad, D. (2015). Principais itens para relatar revisões sistemáticas e meta-análises: a recomendação PRISMA. Epidemiologia e serviços de saúde, 24(2), 335-342. https://dx.doi.org/10.5123/S167 9-49742015000200017

Garcia-Muiña, F. E.; González-Sánchez, R.; Ferrari, A. M.; Volpi, L.; Pini, M. \& Settembre-Blundo, D. (2019). Identifying the equilibrium point between sustainability goals and circular economy practices in an Industry 4.0 manufacturing context using eco-design. Social sciences, 8, 241. http://dx.doi.org/10.3390/socsci8080241 
García-Muiña, F. E.; Medina-Salgado, M. S.; Ferrari, A. M. \& Cucchi, M. (2020). Sustainability transition in industry 4.0 and smart manufacturing with the triple-layered business model canvas. Sustainability, 12, 2364. http://dx .doi.org/10.3390/su12062364

Hizam-Hanafiah, M. \& Soomro, M.A. (2021). The situation of technology companies in industry 4.0 and the open innovation. Journal of open innovation: technology, market, and complexity, 7, 34. https://doi.org/10.3390/joit mc7010034

Horbach, J. (2005). Indicator systems for sustainable innovation. Heidelberg, Germany: Physica Verlag.

Ivascu, L. (2020). Measuring the implications of sustainable manufacturing in the context of Industry 4.0. Processes, 8, 585. http://dx.doi.org/10.3390/pr8050585

Kamble, S. S.; Gunasekaran, A. \& Gawankar, S. A. (2018). Sustainable Industry 4.0 framework: A systematic literature review identifying the current trends and future perspectives. Process safety and environmental protection, 117, 408-425. https://doi.org/10.1016/j.psep.2018.05.009

Keivanpour, S. \& Kadi, D. A. (2019). Internet of things enabled real-time sustainable end-of-Life product recovery. IFAC-PapersOnLine, 52, 796-801. http://dx.doi.org/10.1016/j.ifacol.2019.11.213

Kristoffersen, E.; Blomsma, F.; Mikalef, P. \& Li, J.(2020). The smart circular economy: A digital-enabled circular strategies framework for manufacturing companies. Journal of business research, 120, 241-261. http://dx.doi.o $\mathrm{rg} / 10.1016 / \mathrm{j} . j$ busres.2020.07.044

Mangas-Vega, A.; Sánchez-Jara, J. M. \& Alonso, A. R. (2018). La autopublicación en las bibliotecas: Revisión sistemática de la literatura. Palabra clave, 7(2), e047. https://doi.org/10.24215/18539912e047

Moktadir, M. A.; Ali, S. M.; Kusi-Sarpong, S. \& Shaikh, M. A. A. (2018). Assessing challenges for implementing Industry 4.0: implications for process safety and environmental protection. Process safety and environmental protection, 117, 730-741, http://dx.doi.org/10.1016/j.psep.2018.04.020

Morrar, R.; Arman, H. \& Mousa, S. (2017). The fourth industrial revolution (Industry 4.0): a social innovation perspective . Technology innovation management review, 7(11), 12-20. http://doi.org/10.22215/timreview/11 17

Müller,J. M.; Kiel, D. \& Voigt, K.-I. (2018). What drives the implementation of industry 4.0? The role of opportunities and challenges in the context of sustainability. Sustainability, 10, 247. http://dx.doi.org/10.3390/su10010247

Nardo, M. D.; Forino, D. \& Murino, T. (2020). The evolution of man-machine interaction: The role of human in Industry 4.0 paradigm. Production \& manufacturing research, 8, 20-34. http://dx.doi.org/10.1080/21693277 .2020 .1737592

Oláh, J.; Aburumman, N.; Popp, J.; Khan, M. A.; Haddad, H. \& Kitukutha, N. (2020). Impact of Industry 4.0 on environmental sustainability. Sustainability, 12, 4674. http://dx.doi.org/10.3390/su12114674

Oluyisola, O. E.; Sgarbossa, F. \& Strandhagen, J.O. (2020). Smart production planning and control: Concept, usecases and sustainability Implications. Sustainability, 12, 3791. http://dx.doi.org/10.3390/su12093791http://d x.doi.org/10.3390/su12093791

Ordieres-Meré, J.; Prieto Remón, T. \& Rubio, J. (2020). Digitalization: An opportunity for contributing to sustainability from knowledge creation. Sustainability, 12, 1460. http://dx.doi.org/10.3390/su12041460

Pham, T. T.; Kuo, T.-C.; Tseng, M.-L.; Tan, R. R.; Tan, K., Ika, D.S. \& Lin, C.J. (2019). Industry 4.0 to accelerate the circular economy: A case study of electric scooter sharing. Sustainability, 11, 6661. http://dx.doi.org/10.3 $390 /$ su 11236661

Pimenta, M. L. (2019). Cross - Functional integration in product development processes in the era of industry 4.0. Revista produção e desenvolvimento, 5(2), 350. http://dx.doi.org/10.32358/rpd.2019.v5.350

Ramírez, R. (2007). Calidad de vida relacionada con la salud como medida de resultados en salud: revisión sistemática de la literatura. Revista colombiana de cardiología, 14(4), 207-222. Recuperado de http://www.scielo.org.co/sci elo.php?script $=$ sci_arttext\&pid $=$ S0120-56332007000400004

Requeijo, J. F. G.; Abreu, A. J.; Calado, J. M. F. \& Dias, A. S. M. da E. (2018). Six sigma business scorecard approach to support maintenance projects in a collaborative context. Revista produção e desenvolvimento, 4(1), 82-97. ht tp://dx.doi.org/10.32358/rpd.2018.v4.313 
Rodrigues, A. S. M.; Mello, J. A. V. B. \& Afonso, C. A. G. A. (2019). Desenvolvimiento estimulado por empreendedorismo em incubadoras de empresa: Uma revisão sistemática. MÉI: Métodos de información, 10(19), 1-27. http://dx.doi.org/10.5557 / IIMEI10-N19-001027

Sartori, S.; Lat rônico, F. \& Campos, L. M. S. (2014). Sustainability and sustainable development: A taxonomy in the field of literature. Ambiente \& sociedade, 17(1), 01-22. http://dx.doi.org/10.1590/1809-44220003490

Saucedo-Martínez, J. A.; Pérez-Lara, M.; Marmolejo-Saucedo, J. A.; Salais-Fierro, T. E. \& Vasant, P. (2018). Industry 4.0 framework for management and operations: a review. Journal of ambient intelligence and bumanized computing, 9, 789-801. https://doi.org/10.1007/s12652-017-0533-1

Sima, V.; Gheorghe, I. G.; Subić, J. \& Nancu, D. (2020). Influences of the industry 4.0 revolution on the human capital development and consumer behavior: A systematic review. Sustainability, 12(10), 4035. https://doi.org/10.33 $90 /$ su 12104035

Sony, M. \& Naik, S. (2020). Industry 4.0 integration with socio-technical systems theory: A systematic review and proposed theoretical model. Technology in society, 61, 101248. https://doi.org/10.1016/j.techsoc.2020.101248

Varela, L.; Araújo, A., Ávila, P.; Castro, H. \& Putnik, G. (2019). Evaluation of the relation between lean Manufacturing, industry 4.0, and sustainability. Sustainability, 11, 1439. http://dx.doi.org/10.3390/su110514 39

Waibel, M. W.; Steenkamp, L. P.; Moloko, N. \& Oosthuizen, G.A. (2017). Investigating the effects of smart production systems on sustainability elements. Procedia manufacturing, 8, 731-737. http://dx.doi.org/10.101 6/j.promfg.2017.02.094

Wang, S.; Wan, J.; Li, D. \& Zhang, C. (2016). Implementing smart factory of industrie 4.0: An outlook. International journal of distributed sensor networks, 12,3159805. http://dx.doi.org/10.1155/2016/3159805 